\title{
Efecto de los gradientes de pastoreo ovino sobre la vegetación y el suelo en Península Valdés, Patagonia, Argentina
}

\author{
Germán H. Cheli ${ }^{12,1}$; ; Gustavo E. Pazos ${ }^{1,2}$; Gustavo E. Flores ${ }^{3}$ \& Juan C. Corley ${ }^{4}$ \\ ${ }^{1}$ Instituto Patagónico para el Estudio de los Ecosistemas Continentales (IPEEC, CCT-CONICET CENPAT). Puerto Madryn, \\ Chubut, Argentina. ${ }^{2}$ Facultad de Ciencias Naturales, Universidad Nacional de la Patagonia San Juan Bosco. Puerto Madryn, \\ Chubut, Argentina. ${ }^{3}$ CONICET, Laboratorio de Entomología, Instituto Argentino de Investigaciones de las Zonas Áridas \\ (IADIZA, CCT-CONICET Mendoza). Mendoza, Argentina. ${ }^{4}$ Grupo de Ecología de Poblaciones de Insectos, INTA EEA
} Bariloche. S. C. de Bariloche, Río Negro, Argentina.

\begin{abstract}
Resumen. La introducción de ganado doméstico provocó modificaciones en la vegetación y en el suelo de la Patagonia extra-andina. Estos cambios alteraron procesos ecosistémicos, aumentaron la desertificación y causaron pérdida de biodiversidad. Es frecuente encontrar gradientes decrecientes de actividad animal partiendo de las aguadas (piósferas), que resultan adecuados para determinar el impacto del pastoreo sobre los ecosistemas. El objetivo de este estudio fue evaluar el efecto de la intensidad de pastoreo ovino a partir de gradientes de piósfera sobre la estructura de la vegetación y el suelo en estepas arbustivas representativas de Península Valdés, Argentina. Mediante modelos lineales generalizados mixtos (GLMM) y técnicas multivariadas se estudiaron las variaciones en la cobertura vegetal, complejidad vertical de la vegetación, número y área de parches vegetados, microtopografía del suelo y su compactación en relación con el gradiente de pastoreo. La intensidad del pastoreo disminuyó con la distancia a la aguada y explicó más de $50 \%$ de la variabilidad ambiental. Esto demuestra la existencia de efecto piósfera. La cobertura de herbáceas, mantillo, complejidad vertical de la vegetación y la microtopografía aumentaron al disminuir la presión de pastoreo en los sitios más alejados de la aguada, mientras que el porcentaje de suelo desnudo, gravas, cobertura de anuales y la compactación del suelo presentaron un patrón opuesto. Este estudio evidencia, por primera vez, la existencia de piósferas ovinas en Península Valdés e indica que la intensidad de pastoreo en la región se asocia con alteraciones ambientales compatibles con procesos importantes de degradación ecosistémica. Dada la importancia que reviste la península en el contexto de la conservación biológica de la región, se requieren más estudios de este tipo para una implementación efectiva de medidas de manejo que integren la ganadería y la conservación de los recursos naturales.
\end{abstract}

[Palabras clave: cobertura del suelo, desertificación, estepa arbustiva, ecosistemas áridos, piósfera, presión de pastoreo]

\begin{abstract}
Aвstract. Effect of sheep grazing gradients on vegetation and soil in Peninsula Valdés, Patagonia, Argentina. The introduction of domestic livestock in the arid Patagonia produced changes in vegetation and soil that altered fundamental ecosystem processes, increasing desertification and biodiversity loss. In this region, it is common to observe gradients of decreasing animal activity from watering points where livestock impact is greatest near the watering sites, called piospheres, particularly suitable for assessing the effect of grazing on arid ecosystems. The aim of this study was to evaluate the effect of sheep-grazing intensity using piospheres on the structure of the vegetation and soil in shrubby steppes of Peninsula Valdes, Argentina. Variation of plant cover, vertical complexity of the vegetation, number and area of vegetated patches, micro-topography and soil compaction in relation to piosphere gradients were studied using Generalized Linear Mixed Models (GLMM) and multivariate analyses. Grazing intensity decreased according to increasing distance to the watering point. This fact, explaining more than $50 \%$ of the overall environmental variability, confirmed the existence of a piosphere gradient on each studied site. Such environmental changes were characterized by a gradual increase in grass and litter cover, vertical complexity of vegetation and soil microtopography at increasing distances from the watering point. Percentages of bare soil and gravel, cover of annual grasses and soil compaction showed the opposite pattern. This study demonstrates, for the first time, the existence of sheep piospheres in the shrubby steppes of Peninsula Valdes. Our results indicate that sheep-grazing intensity is associated with changes in the structure and composition of the vegetation and the soil characteristics that are compatible with degradation processes in the study region. Taking into account the importance of Peninsula Valdes in the context of biodiversity conservation, more studies of this type are required to implement effective management actions integrating livestock and conservation efforts on the terrestrial ecosystems of the region.
\end{abstract}

[Keywords: soil cover, desertification, shrub steppe, arid lands, piosphere, grazing pressure]

Editora asociada: Semmartin María

cheli@cenpat-conicet.gob.ar 


\section{INTRODUCCIÓN}

El pastoreo por ganado doméstico es la principal actividad económica en los ecosistemas áridos y semiáridos (Reynolds and Stafford Smith 2002), y su introducción ha producido cambios profundos en la estructura espacial y en el funcionamiento de estos ambientes (e.g., Bisigato et al. 2005; Cingolani et al. 2005). Los efectos del pastoreo sobre la vegetación y los suelos han sido muy estudiados en todo el mundo, y los resultados muestran que el sobrepastoreo es un factor clave en su degradación (Peters and Havstad 2006).

En la Patagonia extra-andina, el ganado ovino fue introducido a principios del siglo $\mathrm{XX}$ con un sistema de pastoreo extensivo organizado en Estancias de entre 10000 y 50000 ha (Ares et al. 2003). Numerosos trabajos han descripto los efectos de este disturbio sobre la vegetación y los suelos en diferentes regiones de la Patagonia (Oliva et al. 1998; Chartier et al. 2011). Esta actividad ha producido cambios importantes en la estructura de la vegetación a múltiples escalas, en particular en el noreste del Chubut. A escala de paisaje se han reportado cambios en la estructura y en el patrón de distribución espacial de los parches de vegetación (i.e., en el área y la densidad de parches), mientras que a escala de comunidad se observa una reducción general en la cobertura vegetal (especialmente de pastos perennes), un incremento de la cobertura de especies menos preferidas y una reducción de la cobertura de costras criptogámicas (Bertiller et al. 2002; Bisigato et al. 2005; Chartier et al. 2011).

El sobrepastoreo (i.e., degradación de la vegetación causada por un exceso de consumo por parte del ganado) también afecta directa e indirectamente la estructura y los recursos del suelo. En particular, la complejidad de la microtopografía (generada por los pastos establecidos, mantillo y rugosidad del suelo) se simplifica debido al pisoteo y muerte de las plantas pastoreadas (Chartier et al. 2011). Este fenómeno altera los patrones de escurrimiento de agua y determina la compactación del suelo y la redistribución y/o la pérdida de sus recursos (Bisigato et al. 2008; Chartier et al. 2011; Rossi and Ares 2012). Este escenario puede desencadenar un proceso de retroalimentación positiva que dificulta el establecimiento de plántulas en los espacios entre parches (Bisigato and Bertiller 2004) e incrementa la exposición del suelo a la erosión hídrica y eólica (Bisigato et al. 2008; Chartier et al. 2011; Rossi and Ares 2012).

Por otra parte, la presión de pastoreo por el ganado dentro de los cuadros (i.e., unidades espaciales de manejo) no es homogénea (Ares et al. 2003). La necesidad de beber regularmente incrementa la intensidad de uso de las áreas cercanas a las fuentes de agua (Lange 1969; Todd 2006). Se ha sugerido que esto conduce al desarrollo de piósferas (i.e., gradientes crecientes de intensidad de pastoreo al disminuir la distancia al agua) (Lange 1969). En el noreste patagónico, la típica provisión de fuentes de agua artificiales (aguadas) únicas dentro de los cuadros de pastoreo favorece el desarrollo de piósferas (Beeskow et al. 1995; Saba et al. 1995; Ares et al. 2003). Estos gradientes ofrecen un diseño experimental adecuado evaluar el efecto del pastoreo sobre los ecosistemas áridos (James et al. 1999; Todd 2006).

El Área Natural Protegida Península Valdés es una de las unidades de conservación de ecosistemas áridos más grande de Argentina. Fue declarada Patrimonio Natural de la Humanidad y Reserva de la Biósfera por UNESCO y según IUCN es un área protegida categoría VI: con manejo sostenible de recursos naturales. Las actividades productivas predominantes son la cría extensiva de ganado ovino y el turismo (Nabte et al. 2013). Las tierras fueron loteadas y vendidas en su totalidad para la explotación de la hacienda lanar en 1901, por lo que el área cuenta con, al menos, 114 años de pastoreo por ganado ovino (Barba Ruiz 2003). A pesar del valor elevado que tiene la Península Valdés para la conservación biológica, la gran mayoría de los estudios que evaluaron el efecto del pastoreo ovino sobre los ecosistemas terrestres del noreste del Chubut fueron realizados fuera de esta área. Los escasos antecedentes que existen (Elissalde and Miravalles 1983; Blanco et al. 2008; Burgi et al. 2012) aportaron evidencia de un efecto negativo del pastoreo ovino sobre la estructura y la composición florística de la vegetación. Sin embargo, los esquemas de muestreo empleados en esos estudios son dispares y no permitieron evaluar el efecto de la intensidad del pastoreo sobre diferentes variables de vegetación y suelos en las estepas arbustivas, la unidad de vegetación predominante en Península Valdés (Bertiller et al. 1980).

El objetivo de este estudio fue evaluar cuantitativamente el efecto de la intensidad 
de pastoreo ovino sobre la estructura de la vegetación y el suelo en estepas arbustivas representativas de Península Valdés. La hipótesis de trabajo propuesta postula que la intensidad de pastoreo ovino disminuye con el incremento de la distancia a las aguadas, desarrollándose piósferas, y que éstas se asocian con cambios en la estructura de la vegetación y en las características del suelo. Se espera que al aumentar la distancia desde la aguada se observe un cambio en variables de vegetación y suelos hacia valores que indiquen una mejor condición de la comunidad vegetal: mayor cobertura de pastos perennes, mantillo y costras criptogámicas, menor cobertura de suelo desnudo y gravas, mayor complejidad estructural de la vegetación, menor compactación del suelo y microtopografía más compleja.

\section{Materiales y Métodos}

\section{Área de estudio}

Península Valdés (PV) comprende una meseta amplia de $\sim 4000 \mathrm{~km}^{2}$, ubicada en el noreste de la Patagonia argentina, provincia de Chubut $\left(42^{\circ} 05^{\prime}-42^{\circ} 53^{\prime} S\right.$; $63^{\circ} 35^{\prime}-65^{\circ} 04^{\prime}$ O). Topográficamente, consiste en una planicie con cordones medanosos en el tercio austral y tres depresiones en el centro y norte del área. El clima es semiárido, con una temperatura media anual de $12.6^{\circ} \mathrm{C}$ y un promedio de precipitaciones de $225 \mathrm{~mm} /$ año, con una variabilidad interanual elevada (Nabte et al. 2013). Los vientos son intensos, predominantemente del sector oeste (Barros and Rodríguez Seró 1981).

En esta región coexisten elementos típicos del Monte y de la Provincia Fitogeográfica Patagónica, por lo que se la define como Ecotono Península Valdés (León et al. 1998). PV presenta un mosaico complejo de comunidades vegetales con diferente fisonomía y composición florística (Bertiller et al. 1980). Este estudio se llevó a cabo en estepas arbustivas características de la porción centro-norte, que representan la unidad de vegetación con mayor superficie en PV (Bertiller et al. 1980) y que se caracterizan por la presencia de los arbustos Chuquiraga avellanedae Lorentz, Chuquiraga erinacea D. Don, Lycium chilense Miers ex Bert., Schinus johnstonii Barkley, Condalia microphylla Cav., y Prosopis denudans Benth., el subarbusto Acantholippia seriphioides (Gray) Mold. y los pastos Nassella tenuis (Phil.) Barkworth, Poa ligularis Nees ex Steud., Pappostipa speciosa (Trin. and Rupr.) Romasch. y Piptochaetium napostaense (Speg.) Hack. Como en otros ecosistemas del noreste del Chubut (Bisigato et al. 2005), la vegetación está distribuida en parches discretos de arbustos y pastos inmersos en una matriz de baja cobertura compuesta por suelo desnudo y pastos dispersos. La actividad ganadera está organizada en establecimientos ganaderos (estancias) divididos en cuadros de $20-25 \mathrm{~km}^{2}$ con una única aguada artificial, que constituyen las unidades de manejo pastoril (Nabte et al. 2013).

\section{Enfoque metodológico y selección de sitios}

Para poner a prueba la hipótesis de trabajo se cuantificaron diferentes variables de la vegetación y del suelo, a distancias crecientes de la aguada dentro de cuadros de pastoreo (Todd 2006) cuyos signos de actividad animal evidenciaban, de forma simultánea e independiente, la ocurrencia de un gradiente de pastoreo. Los cuadros se seleccionaron siguiendo cinco criterios tendientes a minimizar el efecto de factores externos que pudieran afectar a las piósferas (Ares et al. 2003; Todd 2006): 1) los cuadros debían tener una sola aguada artificial y el mismo tipo de ganado ovino, 2) su configuración y la localización de la aguada debía haberse mantenido sin cambios desde la colocación de los alambrados, 3) debían presentar similares tipos de suelo, condiciones climáticas y fisonomía de la vegetación, 4) debían localizarse en diferentes establecimientos ganaderos para garantizar la independencia entre los sitios de muestreo, y 5) no debían tener menos de $5 \mathrm{~km}^{2}$, ni presentar signos de erosión fluvial, ni aguadas naturales, ni otras características topográficas y ambientales que pudieran influir sobre los patrones de actividad de los ovinos. De esta manera se seleccionaron tres cuadros de pastoreo de $\sim 25 \mathrm{~km}^{2}$ de superficie, localizados en las estancias "El Centro" (42 $12^{\prime} 18^{\prime \prime} \mathrm{S}, 63^{\circ} 57^{\prime} 41^{\prime \prime}$

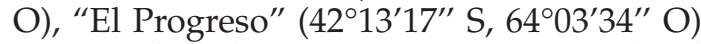
y "La Falsa" (42 $\left.13^{\prime} 30^{\prime \prime} \mathrm{S}, 63^{\circ} 51^{\prime} 45^{\prime \prime} \mathrm{O}\right)$. Al momento de los muestreos, los tres cuadros tenían una carga animal de $16-20$ ovinos $/ \mathrm{km}^{2}$, característica para esta zona de PV (Nabte et al. 2013), y en todos los casos correspondía a majadas de capones y carneros. El régimen de pastoreo era extensivo y continuo a lo largo del año, sin períodos de descanso, como es típico en toda la región.

\section{Diseño y métodos de muestreo}

En cada uno de los cuadros seleccionados se estableció una transecta lineal desde la 
aguada hasta $3200 \mathrm{~m}$ de distancia. Sobre ella se definieron seis sitios de muestreo dispuestos según una escala geométrica a 100, 200,400,800, 1600 y 3200 m de la aguada. Esta disposición incrementa la resolución espacial del muestreo cerca de la aguada (Saba et al. 1995; Todd 2006). A su vez, en cada sitio se definieron tres unidades observacionales dispuestas a lo largo de una línea perpendicular a la transecta de muestreo, separadas entre sí por una distancia mínima de 100 m (Figura 1). En cada unidad observacional se delimitaron dos transectas lineales de $20 \mathrm{~m}$ de longitud, perpendiculares entre sí y con sus puntos medios interceptados (en forma de cruz) (Figura 1). Todo el trabajo de campo fue realizado en el período más seco del verano patagónico (febrero) de 2005, en un lapso de nueve días sin precipitaciones.

Evaluación de la intensidad de pastoreo. La intensidad de pastoreo reciente se evaluó a través de la estimación de la densidad de fecas frescas de ovinos en cada sitio de muestreo (Bisigato and Bertiller 1997). Para ello se registró el número de fecas dentro de cuadros de $0.25 \mathrm{~m}^{2}$ dispuestos cada $2 \mathrm{~m}$ sobre cada una de las transectas de $20 \mathrm{~m}$ (20 estimaciones por unidad observacional) (Figura 1).

Vegetación y cobertura del suelo. Sobre cada una de estas transectas se aplicó el método Point Quadrat Modificado (Passera et al. 1986), utilizando una aguja de $1.5 \mathrm{~m}$ de longitud y $4 \mathrm{~mm}$ de diámetro dispuesta verticalmente cada $25 \mathrm{~cm}$, para estimar la cobertura porcentual de arbustos (especies leñosas con altura máxima superior a $0.8 \mathrm{~m}$ ), subarbustos (leñosas inferiores a $0.8 \mathrm{~m}$ de altura), pastos perennes, herbáceas anuales en pie, mantillo, líquenes, costras criptogámicas, suelo desnudo y gravas. Se registró el número de toques de plantas sobre la aguja en cada punto muestreado para cuantificar la complejidad vertical de la vegetación. A fin de evaluar eventuales cambios en la distribución espacial de la vegetación (i.e., en el tamaño y la densidad de los parches de vegetación) se registró el número de parches de arbustos y subarbustos interceptados por transecta y se midió el diámetro mayor y su perpendicular (en metros) para calcular el área $\left(\mathrm{en}^{2}\right)$ de cada uno con la siguiente formula:

$$
\text { Área parche }=\left(\frac{\text { diam } 1+\operatorname{diam} 2}{4}\right)^{2} * \pi
$$

Microtopografía y compactación del suelo. Para evaluar la microtopografía, en cada extremo de la cruz de cada unidad observacional se midió la longitud relativa de una cadena de $1 \mathrm{~m}$ de longitud con eslabones de $5 \mathrm{~mm}$, dispuesta sobre el sustrato de manera de reproducir su relieve de la manera más exacta posible (Jester and Klik 2005). Luego se calculó el siguiente índice, cuyos valores pueden variar entre 0 (mínima) y 10 (máxima rugosidad):

$$
\text { Microtopografía }=\left(1-\frac{\text { longitud_relativa }}{\text { longitud extendida }}\right) * 10
$$

Finalmente, se estimó la compactación del suelo utilizando un penetrómetro de cono (Davidson 1965). Para ello, en cada extremo de la cruz de cada unidad observacional se seleccionó un espacio abierto entre parches de arbustos y se registró la profundidad de incrustación del penetrómetro luego de tres golpes. La resistencia a la penetración fue expresada en kilopascales $(\mathrm{kPa})$ por medio de la siguiente ecuación (Davidson 1965):

$$
\text { Resistencia }=1541-\left(47^{*} \mathrm{x}\right)
$$

donde $\mathrm{x}=$ centímetros penetrados.

\section{Análisis de datos}

En cada unidad observacional se calculó el valor promedio de cada una de las variables evaluadas (excepto para número de parches, que correspondió al número total de parches interceptados por las dos transectas). De esta manera se obtuvieron tres valores por distancia a la aguada (sitio) y estancia. Estos últimos fueron promediados para cada distancia y estancia en el análisis estadístico.

Respuesta individual de las variables. El efecto de la distancia a la aguada sobre cada una de las variables fue evaluado a partir de modelos lineales generalizados mixtos (GLMM por su denominación en inglés Generalized Linear Mixed Models). La distancia a la aguada fue considerada como efecto fijo, mientras que la identidad de la estancia fue tomada como efecto aleatorio. Se modelaron diferentes distribuciones de la variable respuesta y funciones de enlace en la construcción de los modelos: distribución normal y función identidad para las variables continuas, distribución de Poisson y función logarítmica para los conteos y distribución 

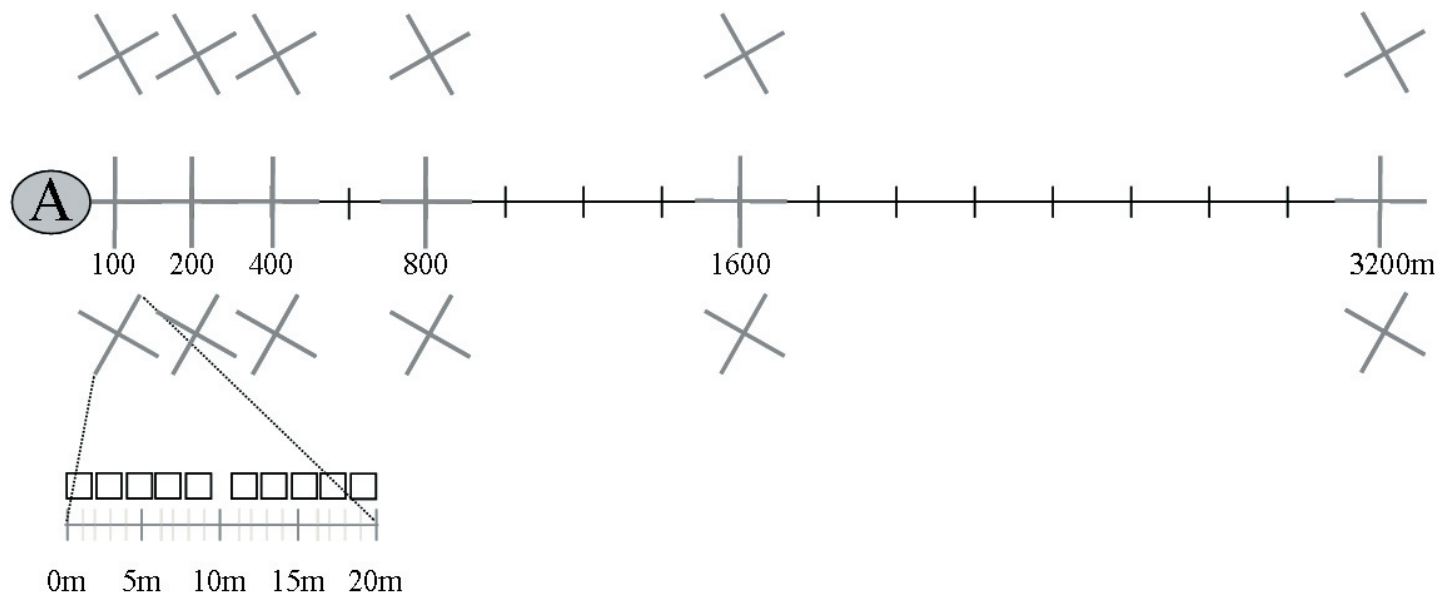

Figura 1. Diseño de muestreo. La línea negra horizontal representa cada una de las tres transectas (una por estancia) de $3200 \mathrm{~m}$ sobre las cuales se dispusieron seis sitios de muestreo a 100, 200, 400, 800, 1600 y $3200 \mathrm{~m}$ de la aguada (A). Las cruces grises representan las unidades observacionales, que consistieron en dos transectas perpendiculares de 20 $\mathrm{m}$, sobre las que se realizaron los muestreos. En la porción inferior se ilustra la disposición de las parcelas de $0.25 \mathrm{~m}^{2}$ (cuadros negros) para cuantificar la densidad de fecas.

Figure 1. Sampling design. The horizontal black line represents each of the three $3200-\mathrm{m}$ transects (one at each Estancia) over which six sampling sites were located at 100, 200, 400, 800, 1600 and $3200 \mathrm{~m}$ from the watering point (A). Grey crosses represent the sampling units consisting of two perpendicular 20-m transects used for sampling. The spatial arrangement of the $0.25 \mathrm{~m}^{2}$ quadrats (black squares) used to quantify the density of sheep faeces is illustrated at the bottom.

binomial y función logit para los porcentajes de cobertura (Tabla 1) (Crawley 2013). Los modelos fueron construidos utilizando modelos de efectos mixtos lineales y no lineales (nlme y lme4) para R (R Development Core Team 2015). La significancia estadística de cada modelo fue evaluada comparando cada uno contra el modelo sin efecto fijo mediante el estadístico $\mathrm{Chi}^{2}$, calculado con la función anova (Crawley 2013). Las pruebas post-hoc en todos los modelos fueron realizadas mediante estadístico $Z$ a partir de la función summary (Crawley 2013).

Variación conjunta de las variables. Dada la naturaleza multivariada de las comunidades vegetales, las técnicas estadísticas univariadas pueden ser inadecuadas para detectar cambios en su estructura (Clarke 1993; Perelman et al. 2005; Borcard et al. 2011). En este sentido, los métodos multivariados, al considerar simultáneamente muchas variables comunitarias, posibilitan la descripción, el análisis y la interpretación de su estructura y de los gradientes ambientales que las afectan (Clarke 1993; Landsberg et al. 2003; Perelman et al. 2005). Por ello, se realizó un Análisis de Componentes Principales (ACP) para revelar la estructura de correlaciones entre las variables evaluadas y los sitios de muestreo. Luego, la variación conjunta en la estructura de la vegetación y del suelo en relación con la distancia a la aguada fue evaluada utilizando Escalamientos Multidimensionales No-métricos (MDS) (Oksanen 2015). La significancia de estos cambios, considerando las estancias como factor de clasificación (bloques aleatorizados), fue evaluada mediante un Análisis Permutacional de la Varianza usando Matrices de Distancia (PERMANOVA) (Anderson 2001). De este modo, las aleatorizaciones sólo ocurrieron dentro de cada una de las distancias y no entre ellas (Oksanen et al. 2015). Finalmente, para evidenciar de forma gráfica estas diferencias se calcularon elipses al 95\% de confianza para los sitios ubicados a cada una de las distancias a la aguada. Si estas elipses se superponen, hay una probabilidad mayor a $5 \%$ de que éstas no sean significativamente diferentes entre sí (Murdoch and Chow 2007). Todos los análisis fueron realizados utilizando el paquete vegan para R (Oksanen et al. 2015).

\section{RESULTADOS}

\section{Evaluación de la intensidad de pastoreo}

La densidad de fecas ovinas disminuyó significativamente al aumentar la distancia a la aguada (Tabla 1). La mayor densidad ocurrió a los $100 \mathrm{~m}$, con 163 fecas $/ \mathrm{m}^{2}$, se registró una zona de alta densidad hasta los $800 \mathrm{~m}$ y desde allí decayó de manera significativa con el aumento de la distancia (1600 y 3200 m) (Tabla 1). 
Tabla 1. Comparación de las variables entre distancias a la aguada mediante GLMM. Se presenta el estadístico $X^{2}$ del cambio en la devianza con relación al modelo nulo y su valor $P$ (g.l.=5; ver detalles en Materiales y Métodos). Se indica valor medio y error estándar (entre paréntesis) de cada variable a cada distancia: densidad de fecas ovinas (Fe); coberturas de arbustos (Arb), subarbustos (Sub), pastos perennes (Pa), herbáceas anuales (An), mantillo (Ma), costras criptogámicas (Ccr), suelo desnudo (Sd), gravas $(\mathrm{Gr})$ y líquenes $(\mathrm{Li})$; número de toques de plantas $(\mathrm{Nt})$ y parches de vegetación ( $\mathrm{Np}$ ) por unidad observacional; área de los parches (Ap); compactación del suelo (Comp) y microtopografía (Mit). Letras diferentes indican diferencias significativas entre distancias.

Table 1. Comparison of the variables among distances to the watering point using GLMM. $\mathrm{X}^{2}$ statistics and its $P$ value (d.f. $=5$ ) for the change in deviance in relation to the null model are presented. Mean value and standard error (between brackets) are indicated for each variable at every distance: density of faeces (Fe); cover of shrubs (Arb), dwarf shrubs (Sub), perennial grasses (Pa), herbaceous annuals (An), litter (Ma), cryptogamic soil crusts (Ccr), bare soil (Sd), gravels (Gr) and lichens (Li); number of plant interceptions (Nt) and shrub patches (Np) per sampling unit; shrubpatch area (Ap); soil compaction (Comp) and microtopography (Mit). Different letters indicate significant differences among distances.

\begin{tabular}{|c|c|c|c|c|c|c|c|c|}
\hline \multirow[t]{2}{*}{ Variable $^{*}$} & \multirow[t]{2}{*}{$x^{2}$} & \multirow[t]{2}{*}{$P$} & \multicolumn{6}{|c|}{ Distancia a la aguada (m) } \\
\hline & & & 100 & 200 & 400 & 800 & 1600 & 3200 \\
\hline $\mathrm{Fe}\left(\mathrm{nro} / \mathrm{m}^{2}\right)^{\&}$ & 57.36 & $<0.001$ & 163.5 & 128.8 & 117.7 & 127.3 & 86.8 & 47.1 \\
\hline $\operatorname{Arb}(\%)^{\#}$ & 8.73 & 0.120 & $\begin{array}{l}(17.4) \mathrm{d} \\
24.4\end{array}$ & $\begin{array}{l}(12.5) \mathrm{cd} \\
23.4\end{array}$ & $\begin{array}{l}(9.2) b c \\
27.6\end{array}$ & $\begin{array}{l}(22.2) \mathrm{c} \\
32.6\end{array}$ & $\begin{array}{l}(12.6) \mathrm{b} \\
30.1\end{array}$ & $\begin{array}{l}(6.5) \mathrm{a} \\
31.2\end{array}$ \\
\hline Sub $(\%)^{\#}$ & 4.61 & 0.465 & $\begin{array}{l}(3.2) \\
0.16\end{array}$ & $\begin{array}{c}(2.1) \\
0.3\end{array}$ & $\begin{array}{l}(2.6) \\
0.43\end{array}$ & $\begin{array}{l}(3.5) \\
0.83\end{array}$ & $\begin{array}{l}(2.8) \\
0.00\end{array}$ & $\begin{array}{l}(2.1) \\
0.46\end{array}$ \\
\hline $\mathrm{Pa}(\%)^{\#}$ & 19.47 & $<0.001$ & $\begin{array}{l}(0.08) \\
10.9\end{array}$ & $\begin{array}{l}(0.15) \\
10.2\end{array}$ & $\begin{array}{l}(0.08) \\
13.3\end{array}$ & $\begin{array}{l}(0.53) \\
19.8\end{array}$ & $\begin{array}{l}(0.00) \\
18.3\end{array}$ & $\begin{array}{c}(0.29) \\
16.8\end{array}$ \\
\hline An $(\%)^{\#}$ & 24.03 & $<0.001$ & $\begin{array}{l}(1.9) \mathrm{a} \\
11.2\end{array}$ & $\begin{array}{l}(3.5) \mathrm{a} \\
4.0\end{array}$ & $\begin{array}{c}(4.8) \mathrm{ab} \\
4.9\end{array}$ & $\begin{array}{c}(5.8) \mathrm{C} \\
4.9\end{array}$ & $\begin{array}{l}(5.7) \mathrm{bc} \\
5.2\end{array}$ & $\begin{array}{c}(5.0) \mathrm{bc} \\
2.6\end{array}$ \\
\hline $\mathrm{Ma}(\%)^{\#}$ & 68.50 & $<0.001$ & $\begin{array}{l}(2.4) \mathrm{b} \\
39.6\end{array}$ & $\begin{array}{l}(0.6) a \\
31.9\end{array}$ & $\begin{array}{c}(1.8) \mathrm{a} \\
45.4\end{array}$ & $\begin{array}{c}(1.7) a \\
61.9\end{array}$ & $\begin{array}{c}(1.8) a \\
45.9\end{array}$ & $\begin{array}{l}(0.8) \mathrm{a} \\
53.8\end{array}$ \\
\hline $\operatorname{Ccr}(\%)^{\#}$ & 4.77 & 0.445 & $(7.1) \mathrm{b}$ & $\begin{array}{l}(6.1) \mathrm{a} \\
8.8\end{array}$ & $\begin{array}{l}(6.6) \mathrm{b} \\
10.4\end{array}$ & $\begin{array}{l}(6.7) \mathrm{d} \\
10.8\end{array}$ & $\begin{array}{l}(9.2) \mathrm{bc} \\
9.6\end{array}$ & $\begin{array}{l}(3.7) \mathrm{c} \\
12.7\end{array}$ \\
\hline $\mathrm{Sd}^{\#}$ & 49.91 & $<0.001$ & $\begin{array}{l}(2.3) \\
26.6\end{array}$ & $\begin{array}{l}(0.3) \\
24.6\end{array}$ & $\begin{array}{l}(3.2) \\
19.2\end{array}$ & $\begin{array}{l}(2.4) \\
10.3\end{array}$ & $\begin{array}{l}(2.1) \\
11.7\end{array}$ & $\begin{array}{l}(2.2) \\
13.3\end{array}$ \\
\hline Gr $(\%)^{\#}$ & 31.49 & $<0.001$ & $\begin{array}{l}(2.5) \mathrm{d} \\
7.9\end{array}$ & $\begin{array}{l}(2.2) \mathrm{cd} \\
14.2\end{array}$ & $\begin{array}{l}(2.7) b c \\
5.7\end{array}$ & $\begin{array}{l}(3.2) \mathrm{a} \\
4.3\end{array}$ & $\begin{array}{l}(1.8) \mathrm{a} \\
4.0\end{array}$ & $\begin{array}{l}(2.0) \mathrm{ab} \\
5.2\end{array}$ \\
\hline $\operatorname{Li}(\%)^{\#(1)}$ & --- & --- & $\begin{array}{l}(1.9) \mathrm{bc} \\
0.06\end{array}$ & $\begin{array}{l}(3.8) \mathrm{d} \\
0.06 \\
(0.06)\end{array}$ & $\begin{array}{l}(0.2) \mathrm{ab} \\
0.06 \\
(0.06)\end{array}$ & $\begin{array}{l}(1.1) \mathrm{ab} \\
0.06\end{array}$ & $\begin{array}{l}(1.8) \mathrm{a} \\
0.16 \\
(012)\end{array}$ & $\begin{array}{l}(1.2) \mathrm{ab} \\
0.66 \\
\end{array}$ \\
\hline $\mathrm{Nt}$ & 248.21 & $<0.001$ & 103.6 & 78.2 & 89.2 & 121.9 & 176.4 & 203.0 \\
\hline${ }_{\mathrm{Np}}^{(\text {nro./un. obs. })^{\&(2)}}$ & 10.24 & 0.068 & $\begin{array}{l}(11.7) b \\
10.5\end{array}$ & $\begin{array}{l}(21.1) \mathrm{a} \\
12.6\end{array}$ & $\begin{array}{c}\text { (30.7)ab } \\
19.3\end{array}$ & $\begin{array}{l}(47.1) \mathrm{c} \\
17.8\end{array}$ & $\begin{array}{l}(69.5) \mathrm{d} \\
15.3\end{array}$ & $\begin{array}{l}(48.6) \mathrm{e} \\
16.2\end{array}$ \\
\hline $\begin{array}{l}\text { (nro/un. obs. })^{\&(2)} \\
\operatorname{Ap}\left(\mathrm{m}^{2}\right)^{\S}\end{array}$ & 3.06 & 0.691 & $\begin{array}{l}(0.8) \\
1.86\end{array}$ & $\begin{array}{l}(0.9) \\
2.00\end{array}$ & $\begin{array}{l}(4.1) \\
1.83\end{array}$ & $\begin{array}{l}(3.4) \\
2.06\end{array}$ & $\begin{array}{l}(2.8) \\
2.16\end{array}$ & $\begin{array}{l}(3.0) \\
2.03\end{array}$ \\
\hline $\operatorname{Comp}(\mathrm{kPa})^{\S}$ & 21.25 & $<0.001$ & $\begin{array}{l}(0.16) \\
1084.0\end{array}$ & $\begin{array}{l}(0.20) \\
955.4\end{array}$ & $\begin{array}{l}(0.40) \\
1010.3\end{array}$ & $\begin{array}{l}(0.38) \\
985.3\end{array}$ & $\begin{array}{l}(0.03) \\
888.1\end{array}$ & $\begin{array}{l}(0.29) \\
916.1\end{array}$ \\
\hline $\mathrm{Mit}^{\#(3)}$ & 54.43 & $<0.001$ & $\begin{array}{c}(31.3) \mathrm{d} \\
0.25 \\
(0.02) \mathrm{a}\end{array}$ & $\begin{array}{c}(38.6) \mathrm{abc} \\
0.31 \\
(0.04) \mathrm{ab}\end{array}$ & $\begin{array}{c}(45.5) \mathrm{c} \\
0.31 \\
(0.05) \mathrm{ab}\end{array}$ & $\begin{array}{c}(30.8) \mathrm{bc} \\
0.33 \\
(0.04) \mathrm{b}\end{array}$ & $\begin{array}{c}(4.6) \mathrm{a} \\
0.37 \\
(0.02) \mathrm{b}\end{array}$ & $\begin{array}{c}(7.0) \mathrm{ab} \\
0.52 \\
(0.04) \mathrm{c}\end{array}$ \\
\hline
\end{tabular}

${ }^{*}$ Modelo de distribución de la variable respuesta empleado en el GLMM: Poisson ${ }^{\&}$, Binomial $^{\#} \mathrm{y}$ Normals.

(1) No fue posible construir un GLMM por su baja frecuencia (menos de 50\%) y cobertura en los sitios de muestreo.

(2) Un. obs.: unidad observacional (Figura 1). Para Nt corresponde a 160 puntos de muestreo con la aguja. Para Np equivale a $40 \mathrm{~m}$ lineales (ver detalles en Materiales y Métodos).

(3) Escala de 0 a 10 indicando mínima y máxima rugosidad, respectivamente.

\section{Respuesta individual de las variables}

Los distintos componentes de la cobertura vegetal variaron en forma diferente a lo largo del gradiente de pastoreo. Mientras que la cobertura de arbustos y subarbustos no varió significativamente, la cobertura de pastos perennes aumentó de forma significativa a partir de los $800 \mathrm{~m}$. Por su parte, la cobertura de herbáceas anuales fue mayor a $100 \mathrm{~m}$ de la aguada que en el resto de las distancias (Tabla 1). La cobertura de mantillo mostró una tendencia creciente desde la aguada hacia los sitios más lejanos, con un máximo a los 800 $\mathrm{m}$, y la cobertura de costras criptogámicas no varió significativamente. Además, la cobertura de suelo desnudo y gravas presentaron sus 
mayores valores a 100 y $200 \mathrm{~m}$ de la aguada, y disminuyeron significativamente al aumentar la distancia (Tabla 1).

Por su parte, el área y número de parches de arbustos no variaron de manera significativa con la distancia a la aguada. Sin embargo, el número de toques de plantas por unidad observacional (indicador de la complejidad vertical de la vegetación) se incrementó al aumentar la distancia (Tabla 1). La compactación del suelo fue significativamente más alta a los 100 m y más baja a los 1600 m que en el resto de los puntos. Por último, si bien la microtopografía presentó valores bajos de rugosidad (siempre menores a 1), se incrementó su complejidad al aumentar la distancia a la aguada (Tabla 1). Si bien la cobertura de líquenes fue 10 veces mayor a los $3200 \mathrm{~m}$ que en las inmediaciones de la aguada, sus frecuencias fueron muy bajas en todos los sitios de muestreo, lo que imposibilitó su análisis posterior.

\section{Variación conjunta de las variables}

Los dos primeros ejes del ACP explicaron conjuntamente el $61.4 \%$ de la variabilidad entre los sitios de muestreo (Figura 2). Los sitios localizados a 100 y 200 m de la aguada se ubicaron principalmente en el cuadrante de valores negativos del CP1 y positivos del CP2, y se caracterizaron por la mayor cobertura de herbáceas anuales (CP1) y por la mayor densidad de fecas ovinas, de cobertura de suelo desnudo y de gravas y compactación del suelo (CP2). Por otro lado, los sitios más alejados de la aguada (1600 y 3200 m) se ubicaron en su mayoría en el cuadrante opuesto diagonalmente; se caracterizaron por la mayor cobertura de arbustos, mantillo y costras criptogámicas, número de parches, complejidad vertical de la vegetación y microtopografía (CP1 positivo) y la mayor cobertura de pastos perennes y área de los parches (CP2 negativo) (Figura 2). De esta manera, puede identificarse un gradiente de cambio de las condiciones ambientales asociadas con la presión de pastoreo creciente desde el cuadrante inferior derecho al superior izquierdo (Figura 2). Los sitios a 400 y 800 m de la aguada ocuparon posiciones intermedias en este gradiente y se localizaron mayormente en los cuadrantes restantes del ACP, caracterizados por condiciones ambientales dispares asociadas con diferencias entre las estancias. A pesar de ello, los sitios de muestreo de cada estancia se alinearon en la misma dirección y sentido que el gradiente descripto anteriormente (Figura 2).

El Escalamiento Multidimensional No Métrico concordó con el gradiente de intensidad de pastoreo (Figura 3). La distancia a la aguada explicó 55.6\% de la variabilidad ambiental entre los sitios (PERMANOVA: $F_{5,17}=3.003, P=0.002$ ). Las elipses del $95 \%$ de

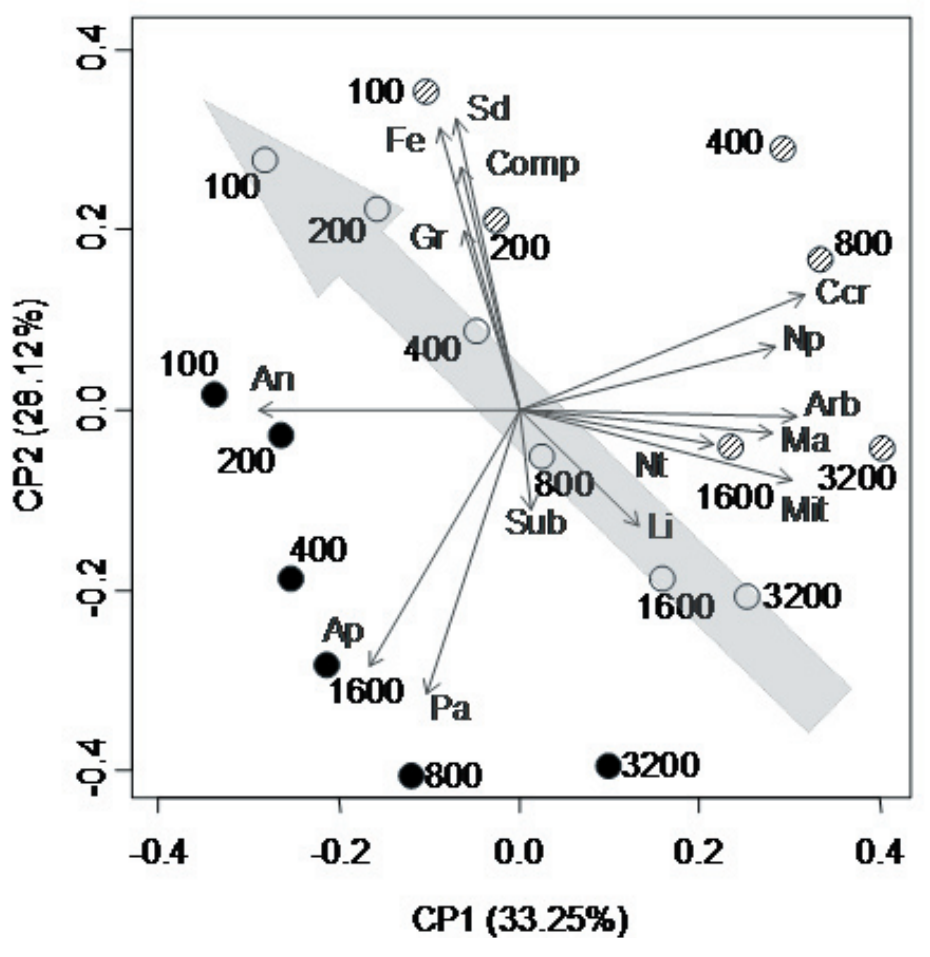

Figura 2. Ordenamiento de los sitios de muestreo (círculos), denotados por la distancia a la aguada (m), y las variables evaluadas (flechas) de acuerdo a los dos primeros ejes del Análisis de Componentes Principales (porcentaje de varianza explicada entre paréntesis). Diferentes colores de los sitios corresponden a diferentes estancias: ø La Falsa; • El Centro; o El Progreso. La flecha punteada gris indica la dirección y sentido del gradiente de presión de pastoreo. Acrónimos para las variables igual que en Tabla 1.

Figure 2. Ordination of sampling sites (circles), labelled with the distance to the watering point $(\mathrm{m})$, and the measured variables (arrows) according to the first two axis of the Principal Component Analysis (percentage of total variance between brackets). Different colours for sites correspond to different estancias: $\varnothing$ La Falsa; • El Centro; o El Progreso. The grey dotted arrow indicate the direction of the gradient of grazing pressure. Acronyms for variables as in Table 1. 


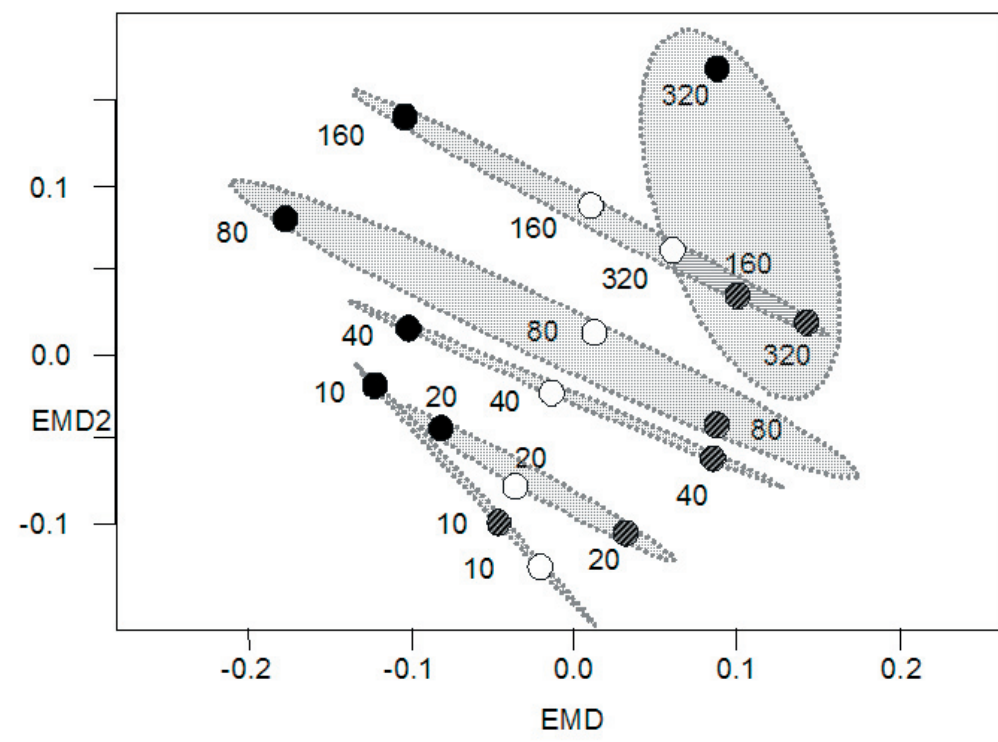

Figura 3. Escalamiento Multidimensional No Métrico de los sitios de muestreo denotados por la distancia a la aguada (m). Las elipses de confianza del 95\% (en gris) definen grupos de sitios con similares condiciones ambientales (elipses solapadas indican diferencias ambientales no significativas entre grupos). Patrón de símbolos igual que en Figura 2.

Figure 3. Non-Metrical Multidimensional Scaling of the sampling sites labelled with the distance to the watering point $(\mathrm{m})$. The $95 \%$ confidence ellipses (in grey) define groups of sites with similar environmental conditions (overlapped ellipses indicate non-significant environmental conditions among groups). Symbol patterns for sites as in Figure 2.

confianza definieron cuatro grupos de sitios en función de la similitud en las condiciones ambientales: 1) los sitios ubicados a 100 y 200 m de la aguada, 2) los sitios localizados a 1600 y $3200 \mathrm{~m}$, que presentaron condiciones disímiles a las del grupo anterior, y los sitios ubicados a 3) $400 \mathrm{~m}$ y 4) $800 \mathrm{~m}$, que si bien evidenciaron heterogeneidad interna, presentaron condiciones ambientales intermedias que las diferenciaron tanto entre sí como de los grupos anteriores (Figura 3).

\section{Discusión}

Este estudio evidencia, por primera vez, la existencia de piósferas ovinas en las unidades de manejo pastoril más comunes de las estepas arbustivas del Área Natural Protegida Península Valdés (cuadros de 25 $\mathrm{km}^{2}$ con una sola aguada). La cantidad de fecas ovinas por metro cuadrado disminuyó al incrementarse la distancia desde la aguada, lo cual evidencia un gradiente decreciente de presión de pastoreo. Estos resultados concuerdan con trabajos previos en otros ecosistemas del noreste patagónico (Bisigato and Bertiller 1997; Bär Lamas et al. 2013). De acuerdo con nuestras predicciones, los sitios más alejados presentaron mayor cobertura de pastos perennes y mantillo, microtopografía y complejidad vertical de la vegetación que los sitios más cercanos, caracterizados a su vez por la mayor cobertura de herbáceas anuales, suelo desnudo, gravas y compactación del suelo.

La mayoría de los cambios en la vegetación y el suelo hallados en este trabajo son consistentes con los reportados para ecosistemas áridos adyacentes a Península Valdés (Bisigato and Bertiller 1997; Bertiller et al. 2002; Chartier et al. 2011) y para otros ecosistemas áridos y semiáridos templados del mundo (James et al. 1999; Landsberg et al. 1999; Morici et al. 2003; Nash et al. 2003; Todd 2006).

La cobertura de pastos perennes se redujo, mientras que la de hierbas anuales se incrementó con el aumento de la presión de pastoreo. La mayor abundancia de hierbas anuales en las áreas más disturbadas cercanas a las aguadas ha sido documentada en un rango amplio de ecosistemas áridos y semiáridos del mundo (Beeskow et al. 1995; Landsberg et al. 1999; Todd 2006; y citas en ellos). Por lo general, este patrón está relacionado con una estrategia de tipo ruderal de estas especies (sensu Grime 1979), las que invierten una proporción elevada de sus recursos en reproducción y ocupan áreas muy disturbadas, donde la intensidad de competencia es menor (Crawley 1998). Por otra parte, estos resultados son consistentes con el hecho de que los pastos perennes constituyen el grupo funcional más preferido por los ovinos (Baldi et al. 2004), y con que su consumo selectivo suele determinar la reducción del vigor y la muerte de las plantas pastoreadas y la disminución del banco de semillas del suelo (Ferraro and Oesterheld 2002; Morici et al. 2003; Pazos and Bertiller 2008). Elissalde y Miravalles (1983) reportaron la presencia de plantas muy consumidas, con los macollos centrales muertos y/o formando pequeños "casquetes" de materia muerta con escasos rebrotes en áreas con evidencia de alta utilización por el ganado. La reducción 
del banco de semillas de los pastos perennes con la presión de pastoreo también ha sido evidenciada en el noreste de Chubut (Pazos and Bertiller 2008). Estos efectos son generalmente más evidentes en las especies de mayor valor forrajero de la región (e.g., Poa ligularis, Nassella tenuis y Piptochaetium napostaense) (Beeskow et al. 1995; Bisigato and Bertiller 1997; Pazos et al. 2007; Burgi et al. 2012). Si bien los relevamientos vegetales de este estudio no se realizaron a nivel específico, es probable que nuestros resultados reflejen mayormente el efecto del pastoreo sobre estas especies, sugiriendo una reducción del potencial productivo del pastizal (Elissalde and Miravalles 1983; Burgi et al. 2012).

La cobertura de arbustos y subarbustos no varió significativamente con la presión de pastoreo. De acuerdo con ello, el área y la densidad de los parches de arbustos tampoco se modificaron significativamente. Esto es, para que la cobertura se mantenga constante, ambas variables deben covariar en sentidos opuestos o mantenerse constantes (Dale 1999). Sin embargo, la complejidad vertical se incrementó conforme disminuyó la intensidad de pastoreo. Estos resultados son consistentes con los de Burgi et al. (2012), quienes no hallaron diferencias en la cobertura de arbustos y subarbustos entre áreas pastoreadas y clausuradas al ganado en el sur de PV. Sin embargo, Beeskow et al. (1995) mostraron un incremento de la cobertura de arbustos en las áreas cercanas a la aguada en un ecosistema similar (Punta Ninfas), atribuido al mayor establecimiento de Chuquiraga avellanedaeen dichas áreas.

Nuestros resultados sugieren además que la presión de pastoreo no modifica el tamaño del grano de heterogeneidad del patrón espacial de parches e interparches en Península Valdés (invariabilidad del tamaño y densidad de los parches de arbustos y subarbustos). En este mismo sentido, Cipriotti y Aguiar (2005) hallaron que la intensidad de pastoreo ovino no modificó el patrón espacial de la vegetación en una estepa arbustiva del sudoeste del Chubut. En contraste, Bisigato et al. (2005) reportaron un incremento en el grano de heterogeneidad de la vegetación con la intensidad de pastoreo para el Monte Austral. Estas diferencias probablemente se relacionen con un efecto diferencial del pastoreo sobre la composición específica de los parches de arbustos en cada región (Bisigato et al. 2005). En relación con ello, en PV podrían esperarse diferencias en la composición florística de dichos parches entre áreas con distinta presión de pastoreo, como ha sido reportado en otros estudios (Beeskow et al. 1995; Bisigato and Bertiller 1997). Por ejemplo, Elissalde y Miravalles (1983) hallaron mayor proporción de especies "deseables" y mayor equitatividad en las áreas con mejor condición general de la vegetación que en aquellas más impactadas por el ganado. Estudios enfocados en el análisis simultáneo del patrón espacial y composición florística de los parches de vegetación son necesarios para explorar estas hipótesis.

La condición superficial y compactación del suelo también variaron con la presión de pastoreo. La cobertura de mantillo fue mínima en los sitios más cercanos a la aguada. Por lo general, el efecto del pastoreo sobre esta variable es mediado por los cambios en la vegetación establecida (Carrera et al. 2008). La menor cobertura de pastos perennes $\mathrm{y}$ el incremento de especies arbustivas siempreverdes con bajas tasas de caída de hojas podrían explicar el patrón observado (Carrera et al. 2008; Campanella and Bertiller 2008). Consistentemente, la cobertura de suelo desnudo y gravas se incrementó a mayor presión de pastoreo, un fenómeno generalizado en ecosistemas áridos y semiáridos (Beeskow et al. 1995; Bisigato and Bertiller 1997; James et al. 1999; Morici et al. 2003). También la microtopografía fue más compleja en los sitios más lejanos a la aguada. La rugosidad del suelo, la presencia de vegetación herbácea y la cobertura de mantillo constituyen barreras al escurrimiento superficial y favorecen la infiltración del agua (Chartier et al. 2011; Rossi and Ares 2012). Los resultados indican que la reducción de la cobertura vegetal más la acción mecánica del pisoteo conducirían a la degradación y simplificación de la microtopografía y al incremento de la compactación. Numerosos estudios han mostrado que estas perturbaciones disminuyen la tasa de infiltración y promueven la redistribución y/o pérdida de recursos del suelo, alterando su estabilidad, funcionamiento hidrológico y ciclado de nutrientes (Nash et al. 2003; Bisigato et al. 2008; Carrera et al. 2008; Chartier et al. 2011).

Los análisis multivariados permitieron relacionar en forma integrada las variables estudiadas con el gradiente de presión de pastoreo. El ACP, el Escalamiento Multidimensional No Métrico y el PERMANOVA permitieron identificar áreas con condiciones ambientales similares a lo 
largo del gradiente de presión de pastoreo, lo cual no hubiese sido posible con el análisis univariado solamente. Las técnicas multivariadas fueron mas eficaces que las univariados en relacionar a la cobertura de arbustos y área de parches con la intensidad de pastoreo debido al efecto estabilizador en la estructura de los datos que genera el considerar simultáneamente varias variables, lo que les brinda mayor habilidad de predicción (Varona et al. 1999) y mayor exactitud a los valores predichos (McGarigal et al. 2000). En este sentido, es importante remarcar la similitud ambiental entre los sitios más cercanos a la aguada (100 y $200 \mathrm{~m})$, caracterizados por la baja cobertura y complejidad vertical de la vegetación perenne, y el suelo más compacto, y entre los más alejados $(1600$ y $3200 \mathrm{~m}$ ), que presentan una mayor cobertura y complejidad de la vegetación perenne, costras criptogámicas y suelo con microtopografía más compleja. Mientras tanto, las áreas ubicadas a distancias intermedias (400 y 800 $\mathrm{m})$ fueron más heterogéneas entre sí, con una combinación de las características antes descriptas y dependientes de la identidad de la estancia. Patrones similares fueron observados en ambientes áridos de Australia (Landsberg et al. 1999) y Sudáfrica (Todd 2006).

Las diferencias entre los establecimientos ganaderos para el rango de distancias intermedias de la aguada $(400-800 \mathrm{~m})$ podrían explicarse a partir de la Hipótesis de Disturbio Intermedio (Connell 1978). Según ésta a lo largo de un gradiente de intensidad de disturbio la variabilidad ambiental se maximiza donde la intensidad del disturbio es intermedia. En ambos extremos del mismo, el ambiente es muy homogéneo ya que donde existe alta intensidad de disturbio muchas especies son eliminadas, mientras que donde la intensidad no es importante como para suprimir la competencia sólo permanecerán los taxones más competitivos.

El uso del hábitat por parte del único ungulado nativo dela región, el guanaco (Lama guanicoe), y su interacción con la actividad ganadera, difícilmente puedan determinar o confundir los patrones observados. Baldi et al. (1997) reportaron una densidad de 0.71 individuos $/ \mathrm{km}^{2}$ para el área de este estudio. Si bien las medidas de protección gubernamentales permitieron restablecer las poblaciones de L. guanicoe, la recuperación se ha dado principalmente en reservas estrictas (Burgi et al. 2012) o en campos dedicados al ecoturismo (Nabte et al. 2013). En este sentido, estos últimos autores reportaron densidades tan bajas como 1.1 individuos/ $\mathrm{km}^{2}$ en las estancias ganaderas. Además, los guanacos pueden saltar libremente los alambrados y utilizar fuentes de agua alternativas. Finalmente, estudios previos han evidenciado que la actividad ganadera ha influido en la distribución espacial de este ungulado, limitándolo a hábitats marginales poco utilizados por los ovinos (Nabte et al. 2013).

Este estudio aporta al conocimiento del estado de los ecosistemas terrestres de PV bajo pastoreo ovino. En un relevamiento que abarcó la mayoría de las unidades de vegetación de PV descriptas por Bertiller et al. (1980), Elissalde y Miravalles (1983) concluyeron que $87 \%$ de los stands relevados presentaban una condición pobre o regular, según un sistema de clasificación de cuatro categorías. Por su parte, en campos de dunas estabilizadas en la región sur del área de estudio, Blanco et al. (2008) identificaron mayor densidad y agregación espacial de hoyos de deflación con el incremento de la presión de pastoreo. Estos hoyos son depresiones formadas por erosión eólica sobre un depósito de arena, y han sido indicados como las estructuras de erosión más comunes en sistemas de dunas en ecosistemas áridos (Blanco et al. 2008). Nuestros resultados indican que en las estepas arbustivas la intensidad de este disturbio está asociado con alteraciones de la estructura y composición de la vegetación (aunque no de su patrón de distribución espacial) y de las características del suelo. Estos cambios son compatibles con fenómenos de degradación ambiental observados frecuentemente en ecosistemas adyacentes a PV (Beeskow et al. 1995; Bertiller et al. 2002; Chartier et al. 2011). La importancia que reviste la región en el contexto de la conservación biológica requiere la profundización de estudios de este tipo para sostener la implementación efectiva de medidas de manejo que integren la ganadería y la conservación (Burgi et al. 2012; Nabte et al. 2013).

Agradecimientos. Los autores agradecen a F. Grandi por la lectura crítica de este trabajo, a A.M. Beeskow por su asistencia botánica, a P. Dell'Arciprete, A. Gómez Laich, A. Liberoff y M. García Asorey por su asesoramiento estadístico, al Centro Nacional Patagónico (CCT-CENPAT CONICET) por facilitar sus instalaciones y logística, a los dueños y puesteros de los establecimientos ganaderos y a la Administradora del Área Natural 
Península Valdés, a la Dirección de Fauna y Flora y Dirección General de Conservación de Áreas Protegidas de la provincia de Chubut por proveer los permisos de muestreo necesarios, a la Fundación Idea Wild por la donación de parte del equipamiento utilizado. Este estudio fue financiado por la Agencia Nacional de Promoción Científica y

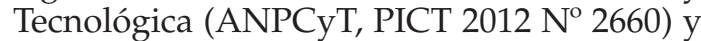
por el Consejo Nacional de Investigaciones Científicas y Técnicas (CONICET, PIP 112201101-00987).

\section{ReFERENCIAS}

Anderson, M. J. 2001. A new method for non-parametric multivariate analysis of variance. Austral Ecol 26:32-46.

Ares, J., M. Bertiller, and A. Bisigato. 2003. Modeling and measurement of structural changes at a landscape scale in dryland areas. Environ Model Assess 8:1-13.

Baldi, R., C. Campagna, and S. Saba. 1997. Abundancia y distribución del guanaco (Lama guanicoe) en el NE del Chubut, Patagonia Argentina. Mastozool Neotrop 4:5-15.

Baldi, R., A. Pelliza-Sbriller, D. Elston, and S. Albon. 2004. High potential for competition between guanacos and sheep in Patagonia. J Wildlife Manage 68:924-938.

Bär Lamas, M., C. Larreguy, A. L. Carrera, and M. B. Bertiller. 2013. Changes in plant cover and functional traits induced by grazing in the arid Patagonian Monte. Acta Oecol 51:66-73.

Barba Ruiz, L. 2003. Acontecimientos Históricos de Península Valdés. Tercera edición. Publicación especial de la Comisión Pro Monumentos a las Gestas y Primeras Colonizaciones Españolas del Chubut. Rawson. Pp. 44.

Barros, V., and J. A. Rodríguez Seró. 1981. Measurements strategies: use of short observations for the annual wind variations. Pp. 3-28 in: International Colloquium on Wind and BHRA Fluvial Engineering.

Beeskow, A. M., N. O. Elissalde, and C. M. Rostagno. 1995. Ecosystem changes associated with grazing intensity on the Punta Ninfas rangelands of Patagonia, Argentina. J Range Manag 48:517-522.

Bertiller, M. B., A. M. Beeskow, and M. D. P. Irisarri. 1980. Caracteres fisonómicos y florísticos de las unidades de vegetación del Chubut. 2. La Península Valdés y el Istmo Carlos Ameghino. Centro Nacional Patagónico - CONICET, Puerto Madryn, Argentina. Pp. 20.

Bertiller, M. B., J. O. Ares, and A. J. Bisigato. 2002. Multiscale indicators of land degradation in the Patagonian Monte, Argentina. Environ Manage 30:704-715.

Bisigato, A. J., and M. B. Bertiller. 1997. Grazing effects on patchy dryland vegetation in northern Patagonia. J Arid Environ 36:639-653.

Bisigato, A. J., and M. B. Bertiller. 2004. Temporal and micro-spatial patterning of seedling establishment. Consequences for patch dynamics in the southern Monte, Argentina. Plant Ecol 174:235-246.

Bisigato, A. J., M. B. Bertiller, J. O. Ares, and G. E. Pazos. 2005. Effect of grazing on plant patterns in arid ecosystems of Patagonian Monte. Ecography 28:561-572.

Bisigato, A. J., R. M. L. Laphitz, and A. L. Carrera. 2008. Non-linear relationships between grazing pressure and conservation of soil resources in Patagonian Monte shrublands. J Arid Environ 72:1464-1475.

Blanco, P. D., C. M. Rostagno, H. F. del Valle, et al. 2008. Grazing impacts in vegetated dune fields: predictions from spatial pattern analysis. Rangeland Ecol Manage 61:194-203.

Borcard, D., F. Gillet, and P. Legendre. 2011. Numerical Ecology with R. Springer, New York. Pp. 306. ISBN 978-14419-7975-9.

Burgi, M. V., A. Marino, M. V. Rodríguez, G. E. Pazos, and R. Baldi. 2012. Response of guanacos to changes in land management in Península Valdés, Argentine Patagonia. Conservation implications. Oryx 46:99-105.

Campanella, M. V., and M. B. Bertiller. 2008. Plant phenology, leaf traits, and leaf litterfall of contrasting life forms in arid Patagonian Monte, Argentina. J Veget Sci 19:75-85.

Carrera, A. L., M. B. Bertiller, and C. Larreguy. 2008. Leaf litterfall, fine-root production, and decomposition in shrublands with different canopy structure induced by grazing in the Patagonian Monte, Argentina. Plant Soil 311:39-50.

Chartier, M., M. Rostagno, and G. E. Pazos. 2011. Effects of soil degradation on infiltration rates in grazed semiarid rangelands of northeastern Patagonia, Argentina. J Arid Environ 75:656-661.

Cingolani, A. M., I. Noy-Meir, and S. Díaz. 2005. Grazing effects on rangeland diversity: a synthesis of contemporary models. Ecol Appl 15:757-773.

Cipriotti, P. A., and M. R. Aguiar. 2005. Effects of grazing on patch structure in a semi-arid two-phase vegetation mosaic. J Veget Sci 16:57-66.

Clarke, K. R. 1993. Non-parametric multivariate analyses of changes in community structure. Austr J Ecol 18:117143.

Connell, J. H. 1978. Diversity in tropical rain forests and coral reefs. Science 199:1302-1310.

Crawley, M. J. 1998. Life history and environment. Pp. 72-131 in: Crawley, M. J. (ed.). Plant Ecology. Blackwell Science Ltd., Oxford.

Crawley, M. J. 2013. The R book. John Wiley \& Sons.

Dale, M. R. T. 1999. Spatial pattern analysis in plant ecology. Cambridge University Press, Cambridge.

Davidson, D. T. 1965. Penetrometer measurements. Pp. 472-484 in: Black, C. A. (ed.). Methods of soil analysis. Part 1. 
Physical and mineralogical properties, including statistics of measurement and sampling. ASA, Madison, Wis.

Elissalde, N. O., and H. R. Miravalles. 1983. Evaluación de los campos de pastoreo de Península Valdés. 70, Centro Nacional Patagónico (CONICET), Puerto Madryn, Argentina.

Ferraro, D. O., and M. Oesterheld. 2002. Effect of defoliation on grass growth. A quantitative review. Oikos 98:125133.

Grime, J. P. 1979. Plant Strategies and Vegetation Processes. John Wiley \& Sons.

James, C. D., J. Landsberg, and S. R. Morton. 1999. Provision of watering points in the Australian arid zone: a review of effects on biota. J Arid Environ 41:87-121.

Jester, W., and A. Klik. 2005. Soil surface roughness measurement - methods, applicability, and surface representation. Catena 64:174-192.

Landsberg, J., S. Lavorel, and J. Stol. 1999. Grazing response groups among understorey plants in arid rangelands. J Veget Sci 10:683-696.

Landsberg, J., C. D. James, S. R. Morton, W. J. Müller, and J. Stol. 2003. Abundance and composition of plant species along grazing gradients in Australian rangelands. J Appl Ecol 40:1008-1024.

Lange, R. T. 1969. The piosphere: sheep track and dung patterns. J Range Manage 22:396-400.

León, R. J. C., D. Bran, M. Collantes, J. M. Paruelo, and A. Soriano. 1998. Grandes unidades de vegetación de la Patagonia extra andina. Ecología Austral 8:125-144.

McGarigal, K. S., S. Stafford, S. Cushman. 2000. Multivariate Statistics for Wildlife and Ecology Research. Springer, New York. Pp. 283.

Morici, E., R. Ernst, A. Kin, D. Estelrich, M. Mazzola, and S. Poey. 2003. Efecto del pastoreo en un pastizal semiárido de Argentina según la distancia a la aguada. Arch Zootec 52:59-66.

Murdoch, D., and E. D. Chow. 2007. Ellipse: Functions for drawing ellipses and ellipse-like confidence regions. R package version 0.3-5.

Nabte, M. J., A. I. Marino, M. V. Rodríguez, A. Monjeau, and S. L. Saba. 2013. Range Management Affects Native Ungulate Populations in Península Valdés, a World Natural Heritage. PLoS ONE 8:e55655.

Nash, M. S., E. Jackson, and W. G. Whitford. 2003. Effects of intense, short-duration grazing on microtopography in a Chihuahuan Desert grassland. J Arid Environ 56:383-393.

Oksanen, J. 2015. Multivariate Analysis of Ecological Communities in R: Vegan Tutorial. http://cc.oulu.fi/ jarioksa/ opetus/metodi/vegantutor.pdf (junio 10, 2015).

Oliva, G., A. Cibils, P. Borrelli, and G. Humano. 1998. Stable states in relation to grazing in Patagonia: a 10-year experimental trial. J Arid Environ 40:113-131.

Passera, C. B., A. D. Dalmasso, and O. Borseto. 1986. Método de “Point Quadrat" modificado. En: Subcomité Asesor del Árido Subtropical Argentino (Ed.). Taller de arbustos forrajeros para zonas áridas y semiáridas. SECYT. Pp. 107.

Pazos, G. E., A. J. Bisigato, and M. B. Bertiller. 2007. Abundance and spatial patterning of coexisting perennial grasses in grazed shrublands of the Patagonian Monte. J Arid Environ 70:316-328.

Pazos, G. E., and M. B. Bertiller. 2008. Spatial patterns of the germinable soil seed bank of coexisting perennial-grass species in grazed shrublands of the Patagonian Monte. Plant Ecol 198:111-120.

Perelman, S. B., W. S. Batista, and R. J. C. León. 2005. El estudio de la heterogeneidad de la vegetación. Fitosociología y técnicas relacionadas. Pp. 321-350 en: M. Oesterheld, M. R. Aguiar, C. M. Ghersa y J. M. Paruelo (eds.). La heterogeneidad de la vegetación de los agroecosistemas. Un homenaje a Rolando León. Editorial Facultad de Agronomía, Buenos Aires.

Peters, D. P. C., and K. M. Havstad. 2006. Non-linear dynamics in arid and semi-arid systems: Interactions among drivers and processess across scales. J Arid Environ 65:196-206.

R Development Core Team. 2015. R: A language and environment for statistical computing. R Foundation for Statistical Computing, Vienna, Austria. http://www.R-project.org/.

Reynolds, J. F., and D. M. Stafford Smith. 2002. Do humans cause deserts? Pp. 1-21 en: J. F. Reynolds and D. M. Stafford Smith (ed.). Global desertification: Do humans cause deserts? Dahlem University Press, Berlin.

Rossi, M. J., and J. O. Ares. 2012. Close Range Stereophotogrammetry and Video Imagery Analyses in Soil Ecohydrology Modelling. The Photogrammetric Record 27:111-126.

Saba, S. L., D. A. Pérez, E. Cejuela, V. Quiroga, and A. Toyos. 1995. La piosfera ovina en el extremo austral del desierto del monte. Naturalia Patagónica, Ciencias biológicas 3:153-174.

Todd, S. W. 2006. Gradients in vegetation cover, structure and species richness of Nama-Karoo shrublands in relation to distance from livestock catering points. J Appl Ecol 43:293-304.

Varona, L., I. Misztal, and J. K. Bertrand. 1999. Threshold-linear versus linear-linear analysis of birth weight and calving ease using an animal model: comparison of models. J Anim Sci 77:2003-2007. 